ISSN 1112-9867

http://www.jfas.info

\title{
NEURO-FUZZY CONTROL OF DATA SENDING IN A MOBILE AD HOC NETWORK
}

\author{
K. O. Polshchykov ${ }^{*}$, S. A. Lazarev, A. D. Zdorovtsov \\ Belgorod State University, 85 Pobeda St, Belgorod, 308015, Russia
}

Published online: 08 August 2017

\begin{abstract}
The article is aimed at data transmission efficiency increase in a mobile ad hoc network, functioning to ensure the exchange of information in emergency situations. The algorithm for neuro-fuzzy control of data sending intensity in this network is proposed. The algorithm provides the measurement of latency period confirmation and the calculation of a moving average of this value, as well as a periodic recalculation of an inter-packet gap by performing neuro-fuzzy inference procedure. By using the algorithm an adaptive change of data sending intensity is provided in the framework of the established logical connection. Simulation experiments showed that the use of the proposed algorithm reduces the number of retransmissions and the average transmission time of data streams in a test network.
\end{abstract}

Keywords: mobile ad hoc network, emergencies, the intensity of data sending, algorithm.

\section{INTRODUCTION}

To ensure the connection within the conditions of intensive external destructive influences specific to natural and man-made emergency situations, telecommunication networks are demanded with a rapid deployment, high tenacity, the ability to deliver information in a dynamically changing topology (random processes of movement, destruction, addition, the turning on and turning off of nodes). Mobile ad hoc networks (MANET) have the specified abilities [1-3].

Author Correspondence, e-mail: polshchikov@bsu.edu.ru

doi: http://dx.doi.org/10.4314/jfas.v9i2s.856 
Due to the specified advantages MANET technology has good application prospects for the development of a mobile ad hoc network for special purpose - the telecommunication network, functioning in order to ensure the exchange of information during the performance of specific tasks related to emergency management. The issues of MANET development for communication provision within the conditions that threaten the lives of people are presented in a series of scientific studies [4-6]. In the future, in order to eliminate the emergency situations it is advisable to use robotic means actively. Scientists have proposed a number of developments in which MANET networks are used to establish the communication between robotic systems in emergency cases $[7 ; 8]$.

However, as experience shows, the presence of stated benefits concerning MANET technology, does not ensure an effective delivery of information supply to subscribers $[9 ; 10]$. The delivery of data in MANET complicates the following main factors:

1) short duration of a connection as the result of network topology rapid changes;

2) a high probability of information distortion due to the interference impact in radio channels;

3) significant packet delivery delay caused by low bandwidth of radio channels;

4) significant packet loss as a result of network congestions which occur frequently due to its traffic unsteadiness.

These factors contribute to a significant reduction of data delivery efficiency to MANET. As it is known, it is required to increase the network performance in order to increase this value. The analysis of the known scientific and technological approaches, aimed at the telecommunications network productivity increase, showed that time interval between packet sending by a source node (interpacket gap) plays an important role in the protocols of a transport layer. By selecting this parameter values, the control of data streams sending intensity by source-nodes is performed.

In existing packet telecommunication networks the control of data sending intensity is based on the TCP (Transmission Control Protocol). According to this protocol an adaptive congestion window selection is performed in the process of data sending by a source node using slow start algorithms, the overload prevention algorithm, a multiplier reset and fast retransmission algorithm [11]. The use of these algorithms leads to the appearance of a network traffic significant fluctuations, thereby the losses of information units (segments) are increased and the data sending is slowed down [12].

In order to implement a theoretically based control of data sending intensity to MANET it is proposed to carry out on the basis of a hybrid neuro-fuzzy intelligent system that combines 
the advantages of fuzzy logic and neural networks [13-18]. The following values are provided to this system input [19-22]:

1) $\bar{M}$ - the current value of the moving average confirmation waiting time;

2) $\bar{M}^{p r}$ - the previous value of the moving average confirmation waiting time;

3) $\tau^{p r}$ - the previous value of an inter-packet interval.

The output value of the system is the variable $\tau$ - the desired value of an inter-packet interval you want to set in a source node during the sending of a next packet. This value is determined as the result of a neuro-fuzzy inference procedure.

The selection of neuro-fuzzy system parameters, the peculiarities of its configuration and operation are presented in [13]. This article is devoted to the development of data sending intensity control algorithm to MANET based on a synthetic neuro-fuzzy system.

\section{Algorithm development}

The process of data sending intensity control to MANET includes the following steps:

1) the measuring of $M$ current values - the confirmation waiting time;

2) the calculation of $\bar{M}$ value;

3) the feeding of $\bar{M}^{p r}, \bar{M}$ and $\tau^{p r}$ to the neuro-fuzzy system of variables;

4) the determination of the output value $\tau$ based on the performance of the neurofuzzy inference, including fuzzification, aggregation, normalization, defuzzification and activation;

5) the setup on a source node when a next packet of the calculated inter-packet interval $\tau$ is being sent.

In order to implement the specified steps the control algorithm concerning the intensity of data sending to MANET is developed, the block diagram of which is presented on Fig. 1.

According to the proposed algorithm, the current value of an inter-packet interval within the established logical connection is periodically updated. The recalculation cycle period of this value makes $I \Delta t$, where $I$ is the number of beats in a cycle, $\Delta t-$ a beat duration. 


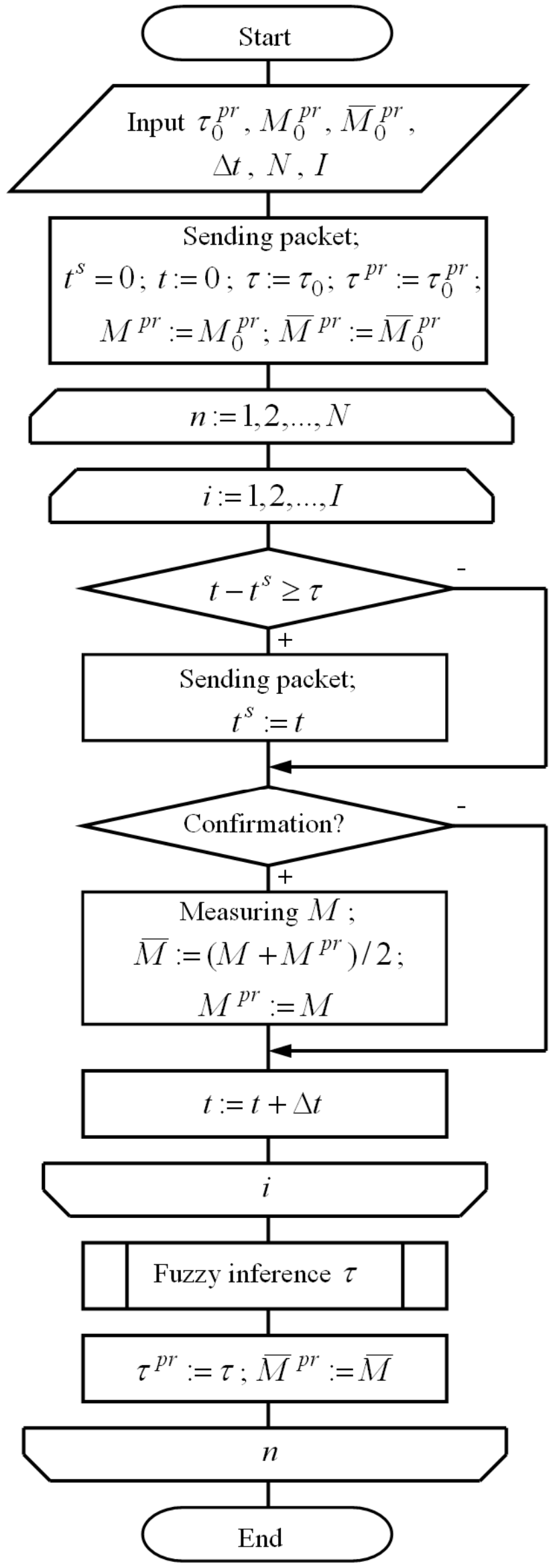

Fig.1. Algorithm block diagram 
The entering of the following values is performed at the beginning of the algorithm: $\tau_{0}^{p r}$ original values $\tau^{p r}, M_{0}^{p r}-$ начального значения предыдущего времени ожидания подтверждения, $\bar{M}_{0}^{p r}$ - the initial value of $\bar{M}^{p r}, N$ - the number of $\tau$ update cycles, and also $I$ and $\Delta t$ update cycles.

Then, during the current moment of time $t=0$ within the established logical connection the first packet sending is performed by the source. At that the moment of packet $t^{s}=0$ is preserved, and also the original values of $\tau, \tau^{p r}, M^{p r}$ and $\bar{M}^{p r}$ are established.

Then the cyclic recalculation of inter-packet interval value is performed, at that $n$ is the number of cycle, changing with the step 1 . The execution of I elementary beats with the duration $\Delta t$ is organized within each cycle. The following operations are performed during each beat:

1) The verification of sending need in a current beat of the next package:

$$
t-t^{s} \geq \tau
$$

2) if the condition (1) is carried out the sending of the next packet by the source is performed, as well as the value of its sending period $t^{s}=t$;

3 ) if the condition (1) is not satisfied, then the next package is not sent by the source in a current beat;

4) the confirmation to the previously sent packet is verified;

5) the measured value $M$ is recorded in case of confirmation within the current beat, the calculation of shifting average waiting time is carried out according to the formula:

$$
\bar{M}=\frac{M+M^{p r}}{2},
$$

and the current value $M$ is given to $M^{p r}$.

At the end of the beat the current discrete time value increases by the value of $\Delta t$.

At the end of the beat the current discrete time value increases by the value of.

After the performance of I beats the procedure of neuro-fuzzy inference value $\tau$ takes place, i.e. the recalculation of inter-packet interval value $\tau^{p r}$ and $\bar{M}^{p r}$ is performed.

In order to assess the effectiveness of the specified algorithm use the simulation model of information flows transmission in MANET was applied created in MatLab \& Simulink software environment [10]. Thus it was stated that the use of neuro-fuzzy control by data 
sending intensity reduces the number of retransmissions by $5.4 \%-11.7 \%$ and the reduction of the average time of data stream transmissions makes $7.2 \%-12.6 \%$.

\section{CONCLUSION}

The increase of data transmission efficiency in a mobile ad hoc network, functioning to ensure the data exchange in emergency situations, is an actual scientific and technical task. The algorithm of neuro-fuzzy control concerning the intensity of data sending by source nodes was developed. The main steps of this algorithm are the measurement of confirmation waiting period and the calculation of a moving average of this value, as well as the performance of the neuro-fuzzy inference procedure for the periodic recalculation of a packet interval.

The result of simulation experiments shows that the application of the proposed algorithm provides a significant reduction in the number of retransmissions and the reduction of the average time for data transmission in MANET.

The research is carried out with the financial support of the Ministries of Education and Science of the Russian Federation, project: 2.5681.2017/8.9.

\section{ACKNOWLEDGEMENT}

The studies were carried out under the financial support of the Russian Federation Ministry of Education and Science (the unique identifier of the project is RFMEFI57815X0138).

\section{REFERENCES}

1. Basagni, S., M. Conti, S. Giordano and I. Stojmenovic, 2004. Mobile Ad Hoc Networking. IEEE Press, pp: 461.

2. Polschykov, K., 2012. Functional model of data flows intensity control in the mobile radio network of the special setting. Scientific Herald of the DSEA, $1: 127-135$.

3. Polshchikov, K.A., 2014. About control of data flows intensity in the mobile radio network for special purpose. Belgorod State University Scientific Bulletin, 21(192): 196-201.

4. Anjum, S. S., R. M. Noor and M. H. Anisi, 2015. Survey on MANET Based Communication Scenarios for Search and Rescue Operations. Proc. of 5th International Conference "IT Convergence and Security (ICITCS)", pp: 1-5.

5. Verma, H. and N. Chauhan, 2015. MANET based emergency communication system for natural disasters. Proc. of International Conference «Computing, Communications \& Automation (ICCCA)», pp:480-485. 
6. Cheong, S. H., I. Lee, Y. W. Si and L. H. U, 2011. Lifeline: Emergency Ad Hoc Network. Proc. of 7th International Conference "Computational Intelligence and Security (CIS)", pp: 283-289.

7. Penders, J., L. Alboul and U. Witkowski, 2011. A robot swarm assisting a human firefighter. Advanced Robotics. 25(1-2), 93-117.

8. Kulla, E., R. Ozaki, A. Uejima and H. Shimada, 2015. Real World Emergency Scenario Using MANET in Indoor Environment: Experimental Data. Proc. of 7th International Conference "Computational Intelligence and Security (CIS)", pp: 336-341.

9. Konstantinov, I., Polshchykov K. and Lazarev, S., 2017. The Algorithm for Neuro-Fuzzy Controlling the Intensity of Retransmission in a Mobile Ad-Hoc Network. International Journal of Applied Mathematics and Statistics, 56(2): 85-90.

10. Konstantinov, I.S., S.A. Lazarev and K.A. Polshchikov, 2015. Simulation model of information flows transmission in mobile ad-hoc network for special purpose. Belgorod State University Scientific Bulletin, 13(210): 156-163.

11. Allman, M., V. Paxson and E. Blanton. TCP congestions control. RFC 5681. Date Views 01.01.2013 www.faqs.org/rfcs/rfc5681.html.

12. Koucheryavy, Y. A., 2004. Traffic control and quality of service in the Internet. St. Petersburg, Science and Technology, pp: 336.

13. Polshchykov, K. O., 2013. Synthesis of neuro-fuzzy systems of data flows intensity control in mobile ad-hoc network. Proceedings of the 23rd International Crimean Conference "Microwave and Telecommunication Technology (CriMiCo)", pp: 517-518.

14. Polshchykov, K., Y.Zdorenko and M. Masesov, 2015. Neuro-Fuzzy System for Prediction of Telecommunication Channel Load. Proceedings of the Second International Scientific-Practical Conference "Problems of Infocommunications Science and Technology (PIC S\&T)", pp: 33-34.

15. Uskov, A.A. and A. V. Kuzmin, 2004. Intelligent control technology. Artificial neural networks and fuzzy logic. Moscow, Hotline-Telecom, pp: 143.

16. Polshchykov, K.O, Y.M.Zdorenko and Masesov M.O, 2014. Method of telecommunications channel throughput distribution based on linear programming and neuro fuzzy predicting. Elixir International Journal. Network Engineering, 75: 27327-27334.

17. Polshchykov K.O., 2015. Router Load Prediction System Based on Fuzzy Neural Network. Herald of Computer and Information Technologies, 9: 55-60.

18. Polschykov, K., Olexij, S. and Rvachova, N., 2010. The Methodology of Modeling Available for Data Traffic Bandwidth Telecommunications Network," Proceedings of the X 
International Conference "Modern Problems of Radio Engineering, Telecommunications and Computer Science (TCSET)", p: 158.

19. Konstantinov, I., Lazarev, S., Polshchykov, K. and Mihalev O., 2015. Theoretical aspects of evaluation of the corporative portal network traffic management. International Journal of Applied Engineering Research, 10(24): 45691-45696.

20. Konstantinov, I., Polshchykov, K., Lazarev, S. and Polshchykova, O., 2017. Model of Neuro-Fuzzy Prediction of Confirmation Timeout in a Mobile Ad Hoc Network. CEUR Workshop Proceedings. Mathematical and Information Technologies, 1839: 174-186.

21. Konstantinov, I., Polshchykov, K., Lazarev, S. and Polshchykova, O., 2016. The Usage of the Mobile Ad-Hoc Networks in the Construction Industry. Proceedings of the 10th International Conference on Application of Information and Communication Technologies (AICT), pp. 455-457.

22. Rvachova, N., G. Sokol, K. Polschykov and J. Davies, 2015. Selecting the intersegment interval for TCP in Telecomms networks using fuzzy inference system. Proceedings of the Sixth International Conference "2015 Internet Technologies and Applications (ITA)", pp: $256-260$.

\section{How to cite this article:}

Polshchykov K O, Lazarev S A, Zdorovtsov A D. Neuro-fuzzy control of data sending in a mobile ad hoc network. J. Fundam. Appl. Sci., 2017, 9(2S), 1494-1501. 